\title{
Tensiones organizacionales en la implementación de las Reformas Educacionales en el Municipio: El caso de Chile*
}

\author{
Organizational conflicts in the implementation of educational reforms in the city hall: The \\ Chilean Situation
Tensões organizacionais na implementação das Reformas Educacionais no município: o caso do Chile

\section{Giselle Davis Toledo}

Universidad de Talca, Instituto de Investigación y Desarrollo Educacional, 5671200255 - 82616814 , gdavis@utalca.cl

\begin{abstract}
RESUMEN
El artículo aborda la relación entre las tendencias y tensiones del sistema educativo chileno y las dinámicas micro organizacionales de los sistemas educacionales municipales en la implementación de las políticas y programas educacionales. Los resultados indican que la confluencia entre la estructura disociada y centralista del sistema educativo chileno, las dinámicas modernizadoras del Estado, plasmadas en las crecientes exigencias de las políticas y programas educacionales, y los límites de la organización municipal para implementarlas, determina la complejización del entorno organizacional municipal. Los actores reaccionan frente a la complejización con conductas pragmáticas y resistentes a la innovación pedagógica orientadas hacia el resguardo del sistema educativo de las presiones y cambios externos. Esta dinámica es contraproducente con los resultados que se esperan de las políticas y programas educacionales a nivel local.
\end{abstract}

Palabras clave: política educacional, municipio, gobernanza, prácticas sociales.

\begin{abstract}
The article discusses the association between micro social organizational dynamics of local educational systems and the possibilities of the main actors, involved in educative policies and programs, to improve education from a territorial educational project. The results indicate that the convergence between the decoupled and centralized structure of the Chilean educational system, the modernizing dynamics of the State represented in the increasing demands of the educational policies and programs, and the limits of the city hall organization to implement them, determine the increasing complexity of the city hall organizational environment. The participants react towards this increasing complexity with pragmatic and innovation- resisting behaviors geared to protecting the education system from external changes understood as threats. This dynamics goes against the results expected of the local education policies and programs.
\end{abstract}

Key words: educational policy, city hall, governance, social practices.

\section{RESUMO}

Discute-se a relação entre tendências e tensões do sistema educativo chileno e das dinâmicas micro-organizacionais do sistema de ensino municipal na implementação de políticas e programas educacionais. Os resultados indicam que a confluência entre a estrutura dissociada e centralizada do sistema educativo chileno e a dinâmica de modernização do Estado, modelada pela crescente demanda de políticas e programas educacionais e pelos limites da organização

* Este trabajo es parte del Proyecto PSD- 60; Programa Bicentenario, Financiado por el CONICYT y ejecutado por IIDEgdavis@utalca.cl 
municipal para implementá-las determinam a complexidade do ambiente organizacional municipal. Sujeitos da educação reagem à complexidade com comportamentos pragmáticos e resistentes à inovação pedagógica orientada para a segurança do sistema de ensino diante das pressões e transformações externas. Esta dinâmica é contraproducente quando se consideram os resultados esperados das políticas e programas educacionais municipais.

Palavras chave: política educacional, município, governo, práticas sociais.

\section{INTRODUCCIÓN}

Las transformaciones en la organización del Estado iniciadas en los años ochenta tienen una serie de implicancias para los actores del desarrollo quienes, más allá del crecimiento y equilibrio macroeconómico, exigen una evolución en la forma de gobernar. Es decir, provocan cambios a nivel de las prácticas de gobernanza y, más precisamente, de la "buena gobernanza". Se entiende la gobernanza como la capacidad de gobierno y de liderazgo estatal para proveer a la sociedad de una dirección coherente hacia el pleno desarrollo (Whittingham, 2002). La gobernanza en tanto marco ideológico programático que orienta las políticas y programas se objetiva en la gestión, es decir, en el conjunto de bienes, normas y procedimientos que componen el sistema organizacional. No es posible disociarlas ya que de la calidad gobernanza dependen las condiciones de posibilidad y de calidad de las prácticas de gestión. Se consideran elementos constituyentes de la gestión: las condiciones de la infraestructura y la tecnología; los objetivos e instrumentos de planificación de las organizaciones; la dirección, estilos de liderazgo, los modos comunicación humana y el clima organizacional, entre otros (Osborne y Gaebler, 1993). Se espera que la articulación entre la gobernanza y la gestión provean sustentabilidad social y cultural a los sistemas organizacionales asegurando así el logro de los objetivos que se plantean. De la visión, orientación y de la dinámica que caracterice la gestión depende no sólo la calidad de la educación sino que también la capacidad de los estudiantes de lograr un futuro mejor (Cox, 2005:27; OCDE, 2009). Consecuentemente, el artículo estudia la gobernanza municipal a través del análisis de las prácticas de gestión, en la implementación de las iniciativas de desarrollo educativo a partir de la perspectiva de sus funcionarios municipales, con especial énfasis en el ámbito local.

Este enfoque del estudio a nivel local, y específicamente en el Municipio, se debe a que se le perfila como la principal organización dónde confluyen y se implementan las Reformas mejoramiento de la educación pública. En efecto, estudios empíricos recientes en el área de la modernización de los gobiernos locales evidencian que la gobernanza y la gestión municipal son dimensiones asociadas a la variación de los resultados educacionales (Pavez, 2004; Lizama y Paredes, 2006-2008). No obstante, se observa que en el ámbito local el estudio de la gobernanza y particularmente de la "buena gobernanza" suscita interés en el país sólo recientemente en el ámbito de la educación pese a que constituye debilidades y claves para comprender el éxito y fracaso de las políticas y programas educacionales. Informes de la OCDE y otros estudios asocian los logros de calidad del sector a déficits de gestión del Municipio, enfatizando que la gran debilidad de las propuestas de desarrollo del país en educación se encuentra justamente en la etapa de la implementación de las mismas (OCDE, 2004-2010; Donoso, 2009; Monjes-Reyes, 2007). Consecuentemente, a nivel local, se explican los bajos rendimientos de las políticas y programas de desarrollo educativo en función de problemas de consistencia en la gestión municipal. 
Numerosos estudios abordan, a partir de distintos ámbitos temáticos y enfoques teóricos conceptuales, la problemática de la gobernanza municipal (Avellanada, 2009; Firinguetti y Pavez 2008; Larragaña, 1995; Román y Carrasco 2007; Paredes y Lizama, 2006; Raczynski y Salinas, 2006). No obstante, poco se conoce sobre la gobernanza desde la perspectiva cualitativa y menos aún a partir de un enfoque estructural constructivista que enfatiza la comprensión de las prácticas de gobernanza como un objeto contextual, subjetivo y estructural (Bourdieu, 1994, 2001). Lo anterior debido a que la discusión en el mundo occidental, en la cual se inserta Chile, ha estado históricamente centrada en temas administrativos, orientada desde una visión estructural de carácter preferentemente económico, cuyo eje es el sistema institucional: entendido como un ente externo, y en problemáticas tales como la dinámica (eficiencia interna y externa) de la oferta y de la demanda educacional. Se ha tratado el problema de la educación cono un fenómeno neutro más que cómo consecuencia de una política educacional (Atria, 2010). Menos aún se conoce sobre lo que sucede en los gobiernos locales acerca de la relación entre las tendencias modernizadoras de las políticas educacionales, las dinámicas de los contextos organizacionales y las visiones, estrategias y reacciones de los actores al implementarlas.

El vacío dejado por los estudios de los procesos reales de gestión, referidos a la implementación de las iniciativas de mejoramiento educacionales a nivel local, releva la importancia de indagar sobre lo que ocurre en la intersección entre las tendencias modernizadoras de las políticas y programas educacionales y la realidad organizacional del Municipio, a nivel de sus prácticas de gobernanza y los actores que las implementan. Ahora, ¿cuáles son las visiones y cómo reaccionan los agentes municipales frente a esta realidad? En efecto, se aborda el estudio de la gobernanza y gestión local a través del análisis de las prácticas de implementación de las propuestas, considerando la incidencia de las dinámicas educacionales macrosociales que impactan los sistemas educacionales municipales. Igualmente, se busca comprender cómo reaccionan los funcionarios municipales a la dinámica que imprimen las políticas y programas en los sistemas educativo locales y su influencia en la forma en que estas se implementan.

El artículo está organizado en tres partes. En la primera se esbozan los elementos y tensiones que condicionan la asimetría en las oportunidades de mejoramiento educativo que afectan a los sistemas educacionales locales no urbanos. En la segunda parte, se aborda el principal resultado del estudio, es decir, el denominado proceso de complejización del entorno organizacional municipal y sus consecuencias en la predisposición de los actores frente a las Reformas educacionales. Se concluye con una síntesis reflexiva sobre la relación entre las condiciones organizacionales de implementación de las políticas educacionales y las lógicas de los actores locales al implementarlas.

\section{TENSIONES ESTRUCTURALES DEL SISTEMA EDUCATIVO CHILENO Y LA ORGANIZACIÓN MUNICIPAL}

\subsection{CALIDAD DE LA EDUCACIÓN Y OPORTUNIDADES}

\section{TERRITORIALES NEGATIVAS}

El abordaje de la gobernanza municipal sitúa el estudio en el ámbito de las políticas de modernización del Estado, proceso que impacta directamente en las dinámicas 
organizacionales de los gobiernos locales. Es así como el fenómeno de la gobernanza no es políticamente neutro, sino que se inscribe dentro de un ideal de sociedad y de gobierno que se plasma en los objetivos y diseño de las Reformas educacionales. La gobernanza implica la búsqueda de la "buena gobernanza", es decir, de una construcción social, deontológica y político ideológica que depende de las perspectivas desde las cuales se aborda. Por lo mismo, no es posible establecer una definición mínima de la noción; no hay consenso en la literatura sobre los principios rectores ni sobre las condiciones que la determinan. En términos deontológicos, en el ámbito especifico de la educación, el fenómeno de la modernización del Estado y de la educación en el país asocian la gobernanza a ideales tales como: la descentralización; la transparencia la gestión; la participación ciudadana; la sustentabilidad social y cultural y la democratización del Estado (Flacso, 2008).

Pese a la normatividad y relatividad en la definición de la noción misma de "buena gobernanza", no se cuestiona su impacto positivo para el desarrollo ni la relación intrínseca entre la capacidad de gobernar y las condiciones político-institucionales dónde se implementan las iniciativas de desarrollo y de desarrollo educativo.

Existe consenso en el país sobre la necesidad de cambios a nivel del diseño político institucional del sistema educativo para que favorezcan una educación de calidad. De esta forma, la descentralización administrativa y la privatización de los servicios sociales orientan tempranamente en Chile las Reformas educacionales del Estado, de segunda generación en comparación al contexto regional, asociándose a importantes déficits en términos de la calidad y de la equidad del servicio educativo y, por ende, a un ideal de "buena gobernanza" (Hoxby, 2002; Moe, 2001; Wong, 1992; Cepal 2009). Los 23 años de municipalización ${ }^{1}$ o administración por los Municipios ${ }^{2}$ de sus respectivos sistemas educacionales (Rodríguez y Winchester 1994) y el sistema de "voucher" basado en la premisa de la libre elección de escuelas por los padres, que actuaría como incentivo competitivo entre proveedores públicos y privados de educación para captar la matricula vía el mejoramiento del servicio, no logró mejorar la calidad educacional (Hoxby, 2003; Moe, 2001; Wong, 1992; Elaqua y Fabrega 2004; Donoso y Hawes 2002). Contrariamente a lo esperado, las Reformas educacionales implementadas no solamente dejan en status quo los indicadores de calidad de la educación, más bien aumentan las inequidades sociales y territoriales, disparando la brecha entre la inversión y la calidad de la educación entre los Municipios ricos y pobres (Di Gropello, 1999; García-Huidobro y Bellei 2003: 46; Quezada-Hofflinger, 2009). ${ }^{3}$

1 La Reforma Educacional Chilena o municipalización fue el último legado legislativo de la dictadura militar de Augusto Pinochet a la democracia chilena re instaurada tras el golpe de Estado de 1973 en 1989 por los gobiernos de la Concertación de Partidos por la Democracia (1990-2010). Desde esa fecha se mantiene un sistema de educación mixto donde el Estado a través del Ministerio de Educación supervisa a los proveedores públicos (Municipios) y privados de educación.

2 Se debe recordar que los Municipios están definidos como "corporaciones autónomas de derecho público, con personalidad jurídica y patrimonio propio, cuya finalidad es satisfacer las necesidades de la comunidad local y asegurar su participación en el progreso económico, social y cultural de las respectivas comunas”,, Ley Orgánica Constitucional de Municipalidades, Ministeriodel Interior, 2002. enelámbitoterritorial tienen comoresponsabilidades la administración del espacio público, el equipamiento comunal, el tránsito, los servicios de aseo y ornato y la regulación del área urbana. "capacidad de gobernar, ejercer autoridad y realizar acciones que ordenen, dispongan y organicen el territorio contenido en su jurisdicción con el fin de satisfacer las demandas de su población".

3 Según Quezada (2009) los progresos en inversión en educación entre los años 2000 y 2006 de las familias y consecuentemente también en los resultados SIMCE (Cox y Lemaître, 1999) se deben más que al sistema de libre elección vouchers al mejoramiento general de la calidad de vida que han experimentado los habitantes del país. 
Siguiendo la misma línea de análisis queda de manifiesto que la distribución de oportunidades para acceder a una educación de calidad tiene un sesgo territorial negativo. La mayor parte (46\% de 1,8 de estudiantes) de los niños y jóvenes que corresponden al $70 \%$ del País que asisten a escuelas públicas proviene de hogares de escasos recursos (Cit. en Marcel y Raczynski 2009) y tienen un rendimiento escolar más bajo que el promedio nacional. El sistema educativo chileno es altamente segmentado (González et al., 2002; Auguste y Valenzuela en Marcel y Raczynski 2009: 37), situación que afecta de manera notable a los sectores rurales. El 66\% de las escuelas básicas del País que corresponden a un $62 \%$ son de administración municipal y, si bien tienen más participación en el sistema educativo, en cuanto al mayor número de escuela su matrícula, es baja y disminuye progresivamente (2009: 57$)^{4}$.

Es decir, la escuela pública municipal tiende a acoger a los sectores pobres del estudiantado y, pese a la progresiva merma en la matrícula de alumnos el Municipio, sigue siendo uno de los principales proveedores de educación, abarcando cerca del $50 \%$ de los alumnos que asisten a colegios subsidiados por el Estado (Raczynski y Salinas 2007) y a más del $70 \%$ de la población más pobre (Sincé, 2009). Los sistemas educacionales municipales se enfrentan también a problemas de financiamiento, ya que es más caro educar a estudiantes pobres que viven en comunas de pequeñas, de región y rurales (Espínola et al., 1996; Espíndola, 1997; Bellei y González, 2003, Hanushek, 1986, 1995; Gallego, 2006; Mizala et al., 2007; Donoso, 2008). El tipo de población atendida por el sistema educativo público tiene necesidades específicas que no fueron consideradas en las Reformas de primera generación y que se consideran como externalidades del mercado. A esta condición de concentración de la pobreza en el sistema de educación pública se suma el hecho que en sectores rurales, donde sólo hay espacio para un proveedor, se anula el incentivo de la competencia demostrando el mal diseño del sistema tanto técnico como financiero de las Reformas (Bellei y González 2004; Elacqua y Fabrega, 2007; Sapelli, 2003; Sapelli y Vial, 2002).

De esta forma, es un lugar común en la literatura sobre la implementación de las Reformas Educacionales afirmar que los Municipios, pobres, pequeños y rurales, presentan importantes problemas de gestión de sus respectivos sistemas educacionales comunales (Fuentes et al., 2007; Raczynsk y Salinas, 2007; Marcel y Raczynski, 2009; Serrano et al., 2000; Donoso et al., 2010). Pese a que se estima que el interés por la administración del sistema educativo está ganando adeptos (Marcel y Raczynski, 2009) el mejoramiento de la gestión no es prioridad para los gobiernos comunales ni las autoridades educacionales (Gallego y Seebach, 2007). Incluso el Programa de Mejoramiento de Gestión Municipal no tuvo finalmente una orientación definida hacia ese objetivo. Sólo en los Municipios con mayores recursos existirían buenas condiciones para el logro de una gestión municipal $^{5}$ de calidad (Matus et al., 2007:235).

4 La participación de los establecimientos es mayor que la matricula, con 62 alumnos por escuela, contra 424 por escuela urbana para el 2007, para la media es 237 contra 408 en lo urbano. Pero si es importante en términos de cantidad de Municipios establecimientos y docentes. Poco se sabe de su gestión pedagógica, no rinden el SIMCE., la tasa de ecuación de las escuelas rurales es del $51 \%$, bájala matricula en el sector básico municipal, y aun mas en los sectores rurales donde los cabios de matriculas están dominados por sectores demográficos y migratorios municipalidad que por la competencia con el sector particular subvencionado. (Marcel y Raczynski 2009:57-61).

5 Ahora bien, cabe destacar, según ese mismo estudio que esta no es una condición determinante ya que existe una alta dispersión en los resultados educacionales lo que implica que existe espacio para mejoras significativas 
No obstante, por el lado de la gestión es necesario para el éxito de las políticas de mejoramiento educativo ya que, mientras mayor importancia cobra el rol del Municipio como ejecutor de las mismas, sobre todo para los sectores vulnerables, más importancia reviste la calidad de la administración a nivel local. Así, el nuevo escenario de las políticas de desarrollo implica para los Municipios, cuya función histórica en educación se ha reducido escasamente a gestionar una que otra escuela, debe asumir un importante liderazgo y responsabilidad social en materia educacional transformándose en un "facilitador del desarrollo local (IULA/CELCADEL, 1993) y generando estrategias que se traduzcan en acciones que beneficien a la comunidad en su conjunto" (Ebert et al., 2006 Cit. en Castillo, 2007: 104).

En consecuencia, se asume que los contextos macrosociales en los cuales se inscriben la gobernanza y la gestión educacional inciden en su calidad y en la variación de los resultados educacionales. La gestión en tanto modo específico de concreción de las iniciativas educacional determina su éxito y la calidad de vida de la población a la cual se dirigen. La gobernanza y la gestión permiten situarse a nivel de los contextos microsociales dónde se implementan las iniciativas de desarrollo, determinan su calidad y son determinados por éstas. En Chile este contexto se caracteriza por la municipalización y la privatización de la educación instauradas por las Reformas estructurales de primera generación las que no logran resolver satisfactoriamente el problema de la calidad ni de la equidad educacional. Contrariamente, se observa un sesgo territorial negativo en la distribución de oportunidades educacionales. Los Municipios de comunas pequeñas de regiones a los cuales acceden justamente los estudiantes más pobres del país, presentan importante problemas de gestión para la implementación de políticas y programas de mejoramiento educativo.

El problema que enfrentan los Municipios no es menor ya que sobre la organización municipal recae la responsabilidad de demostrar la pertinencia de las Reformas pero se encuentra entrampada entre sus propios problemas de precariedad de la organización y una serie de tensiones estructurales que no le han permitido asumir satisfactoriamente la misión que le fue asignada.

\subsection{TENSIONES DEL SISTEMA EDUCACIONAL Y LOS SISTEMAS EDUCACIONALES LOCALES}

Los problemas de la calidad de la educación se asocian a múltiples tensiones macrosociales que estructuran el sistema educativo y condicionan la capacidad de gobernanza y gestión del Municipio. Kooiman propone definir la gobernanza como "los patrones y estructuras que emergen en un sistema sociopolítico, como el 'común resultado' de los esfuerzos de intervención interactiva de todos los actores implicados" (1993: 258). La gobernanza en tanto proceso microsocial que se realiza en las interacciones entre actores concretos en contextos institucionales específicos está determinada por las lógicas macrosociales y de funcionamiento político institucional del Estado. Bajo esta consideración se observa que los sistemas educacionales locales están supeditados normativamente e institucionalmente al modelo de desarrollo, sistema de gobierno y diseño institucional del sistema educativo nacional. Se asume que las tensiones del modelo de desarrollo,

en las prácticas de gestión de los Municipios, lo que sugiere políticas de traspaso de buenas prácticas entre Municipios, probablemente con incentivos asociados a su implementación (Matus et al. 2007). 
la controversia centralización/descentralización y la disociación del sistema educativo a las dificultades del Municipio, afectan particularmente las oportunidades de los sistemas educacionales de comunas pequeñas de mejoramiento de la educación.

Dentro de las tensiones identificadas asociadas a la organización municipal se funda la controversia político ideológico sobre la pertinencia y capacidad del mercado o del Estado de regular los bienes públicos. Tal como plantea Oliva (2009) la situación remite a un problema ético, jurídico y social sobre el rol y el derecho mismo del Estado. Está en juego la determinación de las fronteras estructurales jurídico normativas y político institucionales del Estado, y del mercado para intervenir en lo que "constituye una buena educación y cómo lograrla para asegurar que los estudiantes tengan la mayor oportunidad de aprender" (OCDE, 2004: 289). En la práctica la "gran reforma liberal educacional" presenta dificultades en conciliar el funcionamiento del sistema de manera que cumpla con los postulados y estándares pedagógicos resultando un estancamiento de los resultados escolares (Donoso y Donoso, 2009). Las Reformas educacionales implementadas a partir de los años ochenta se sitúan, entre dos modelos socioeconómicos de administración de los bienes públicos en tensión, el modelo neoliberal y el de Estado protector (Donoso, 2005; Ocde, 2004). La municipalización, acorde con el espíritu neoliberal, restringe las otrora amplias facultades regulatorias del Ministerio de Educación de intervenir directamente sobre la gestión educacional de sostenedores públicos y privados sin contar con dispositivos regulatorios eficientes que lo sustituyan (Eyzaguirre et al., 2005). En este escenario los gobiernos locales se encuentran sin referentes políticos institucionales públicos con capacidad de avalar y sostener las nuevas funciones de administración de la educación que se le encomiendan.

Una segunda tensión estructural se asocia al hecho de que el sistema educativo "se dice descentralizado, pero su motor es centralizado y obedece a prácticas centralistas de antaño" (Raczynski y Salinas, 2006: 206) y a una lógica más bien desconcentradora de los servicios administrativos. La tensión descentralización/centralización de la educación pública se realiza en el marco de la falta de competencias y de recursos jurídicos institucionales de los gobiernos locales para responder y adaptar las reformas educacionales a las demandas y a los contextos locales comunales y, por ende, también regionales. Y si bien no hay suficiente evidencia empírica en Chile (Marcel y Raczynski 2009) ni entre los países de la OCDE (Mons, 2007) sobre el mejoramiento de los resultados educacionales, vía la descentralización política, se sabe que las buenas experiencias en educación se asocian a prácticas descentralizadoras sustentadas desde y en las capacidades de los gobiernos locales (Marcel y Raczynski, 2009).

Contrariamente, ocurre en el país que la precariedad de la descentralización se manifiesta precisamente en la falta de competencias asignadas al Municipio. Éste no se encuentra preparado para asumir la responsabilidad que el Estado le endosa a partir de las reformas de 1980, como líder y principal responsable de las iniciativas de superación de la pobreza, de mejoramiento de la salud primaria y de la educación. La organización municipal ha estado históricamente centrada en la administración de los servicios básicos e infraestructura del territorio Castro (2007). La descentralización no ha sido más que un proceso de desconcentración administrativa que limita la responsabilidad del Municipio a administrar y operar políticas educacionales unificadas del Ministerio de Educación.

Es así como la tensión descentralización/centralización de la educación pública implica dificultades a nivel municipal para implementar los programas educacionales y 
adaptarlos a las demandas y contextos locales ${ }^{6}$. Esta tensión nos remite directamente a los límites de la organización municipal frente a los procesos de modernización que se plasman en las exigencias crecientes de las políticas educacionales.

La tercera tensión remite al impacto que tiene sobre el Municipio la disociación estructural del sistema educativo del país en cual asigna las funciones técnico pedagógicas, administrativas y operativas a actores institucionalmente separados. En efecto, el sistema educativo chileno no sólo es centralizado, lo que implica en sí mismo al menos un desfase entre las necesidades locales y las planteadas por los gobiernos centrales, sino que además presenta problemas de diseño organizacional entre sus distintos niveles. Las funciones del sistema educativo chileno se reparten entre las técnico-pedagógicas, administrativas y de conducción de las escuelas están asignadas a al Ministerio de Educación, a los Departamentos de Administración Municipal de Educación y a los establecimientos escolares respectivamente (Gallego y Seebach, 2007:79: OCDE, 2004:). Cada unidad se hace cargo de funciones discretas de un proceso complejo sin tener poder real de injerencia en las distintas etapas de la implementación de las políticas y programas de mejoramiento educacional (Castro, 2009).

Las municipalidades operan con una lógica político administrativo y socio-territorial distinta al gobierno regional ocupado en asuntos de inversión regional, principalmente de infraestructura (Serrano, 2001). El Municipio administra los servicios básicos, el sistema educativo, la atención primaria en salud y en general los programas, proyectos y red social del gobierno dirigidos fundamentalmente hacia los grupos vulnerables. La cercanía con la sociedad local lo aventaja en la identificación de las demandas colectivas y el cumplimiento de funciones vinculadas a cuestiones básicas de la vida comunal. No obstante su posición privilegiada respecto a las demandas territoriales existiría un divorcio entre ambos eslabones que deja en el vacío la gestión territorial propiamente tal Castro (2009) y las dimensiones técnico pedagógico de las políticas de mejoramiento educativo ${ }^{7}$.

Esta disociación se amplifica en el contexto centralizado que enmarca las iniciativas educacionales ya que la dinámica jerarquizada del centralismo resta competencias a quienes las implementan. La operatoria actual en la implementación de las políticas y programas educacionales no permite a los funcionarios municipales corregir errores de diseño y rigideces que se revelan al momento de su ejecución. Se sabe también que el centralismo favorece roles y dinámicas pasivas y de irresponsabilidad en la implementación de las propuestas de mejoramiento.

La disociación en el cumplimiento de las funciones del sistema educativo no se agota en su estructura de funcionamiento, pues dentro del sistema municipal mismo los Departamentos de Educación delegan funciones de planificación y programación educacional. Zamorano (2006: 12) documenta al respecto como las deficiencias técnicas de los PADEM $^{8}$, por ejemplo, amplifican la disociación de los objetivos educacionales al interior mismo de los Municipios al asumir la $\operatorname{SECPLAC}^{9}$ la elaboración de los proyectos

$6 \quad$ En Chile existen 341 Municipios (225 son rurales y 116 urbanos) cuya población varía desde 316 a 501.042 habitantes por comuna (Ministerio del Interior, 2002).

7 Se hace referencia en la sección 3.0 a esta situación.

8 Los PADEM (Planes de Desarrollo Educativo Municipal) constituyen la planificación anual, que incluye objetivos y asignación de recursos del sistema educativo comunal.

9 Secretarias de Planificación Comunales, unidades encargadas de la planificación y el diseño de proyectos al interior del Municipio. 
de infra estructura educacional. Situación que opera en una escasa colaboración entre ambas unidades y que significa riesgos en cuanto a la pertinencia de las iniciativas de infraestructura en cuestión.

La centralización favorece problemas de gestión, vinculación, y por ende de coherencia en los objetivos y las prácticas de implementación de las políticas y programas educacionales. Los espacios de coordinación disponibles, por ejemplo, entre el Ministerio de Educación, los sostenedores municipales (administradores del servicio educativo) y las escuelas dependen en gran medida del arbitrio y criterio personal de los actores ya que no se encuentran avaladas por normativas jurídico institucionales. Se trata entonces de acuerdos y coordinaciones frágiles pero no por ellos menos necesarios si se busca asegurar la coherencia de la implementación de las iniciativas de desarrollo educativo.

En síntesis, las lógicas no siempre convergentes entre los modelos de desarrollo sobre el rol del Estado y del mercado, entre la ideología descentralizadora de las políticas y la centralización de facto de los gobiernos locales y la disociación en el funcionamiento del sistema educativo, afectan las modalidades de gestión de los sistemas educacionales municipales. Es decir, el sistema educativo que debería sostener los sistemas educacionales locales para asegurar el éxito de los objetivos de las Reformas educacionales es parte de las dificultades que éstos enfrentan amenazando la coherencia de la organización municipal y deprimiendo las posibilidades de mejoramiento de la educación. Las tensiones antes señaladas se potencian, impactan las dinámicas organizacionales del Municipio, desafían los límites de sus capacidades.

\section{COMPLEJIZACIÓN DEL ENTORNO ORGANIZACIONAL MUNICIPAL}

Los actores municipales coinciden en definir su entorno organizacional, así como las tendencias en las políticas y programas de mejoramiento educativo, como en un proceso incremental de complejización. Se acuña el término complejización para el análisis, por cuanto no sólo ha sido utilizado de manera indirecta -en la literatura para referirse a la gestión local educacional-, sino que constituye una importante categoría inductiva que refleja pertinentemente la multidimensionalidad del fenómeno abordado. De esta forma se le entiende como el paulatino grado de exigencia de las iniciativas de desarrollo. Referido a la visión de los funcionarios municipales, este proceso se asocia a lo que se le denomina la triple tensión que influye en la dinámica organizacional de los sistemas educacionales locales, la cual remite a las contradicciones que se plasman en las políticas educacionales : 1) entre los modelos de Estado benefactor y neoliberal que las inspiran, 2) las tendencias descentralizadoras de las políticas -en un país unitario altamente centralizado- y 3) la disociación en la distribución de funciones del sistema educativo. Estas controversias impactan de manera negativa los sistemas educacionales locales, lo que les resta oportunidades de desarrollo.

\subsection{COMPLEJIZACIÓN DEL ENTORNO ORGANIZACIONAL MUNICIPAL}

Las controversias en el modelo de desarrollo, en las dinámicas centralizadoras/descentralizadoras y la asignación diferenciada de funciones del sistema educativo, influyen en la dinámica del sistema organizacional municipal y son contraproducentes con la calidad 
de las propuestas de mejoramiento educativo. La ordenación municipal se dinamiza y tensiona determinando lo que los actores señalan como la complejización del entorno organizacional municipal.

La siguiente cita representa la tendencia en la lectura de los funcionarios municipales frente a la problemática que afecta a su organización:

Ahora, si entramos en el análisis de las últimas políticas públicas a nosotros nos han ido complicando la administración, nos han ido generando mucho mayor dedicación de tiempo, de experticia, de competencias nuevas que, por un lado, está bien, pero que ocupan mucho tiempo en lo que es instalación y no pensando mas allá en los logros que se puede tener [Participante $N^{o} 19$, funcionario municipal].

Cuando se habla de complejización, no se hace en el sentido de los recientes estudios en economía de la educación que la asocian a la relación entre la capacidad de producir educación de calidad, con una alta cobertura de un modo eficiente y las condiciones socioeconómicas, geográficas, costos de educar en una comuna (Gallego y Seebach 2001; 17; 2007). Ni se hace tampoco en el sentido práctico y comúnmente empleado, que homologa la complejidad a la presencia de un mayor número de variables o elementos presentes en el fenómeno. Como igualmente respecto del sentido de quienes entienden la complejidad del entorno como las características propias y las dinámicas de desarrollo, que afectan los procesos de transformación socio espacial de las comunas (Fuentes, Allard y Orellana, 2007). El análisis de la noción, tal cual ésta se presenta en el discurso de la gente, se acerca más bien a la noción de Boisier (2003). Es decir, a la noción vigente en el desarrollo intelectual contemporáneo que emerge de la voluntad de análisis de las múltiples dimensiones, de la interdisciplinariedad y de la multilinealidad de los fenómenos sociales.

Diversos autores que abordan el estudio de la descentralización en general y educativa en particular, hacen referencia a los cambios e integración de nuevas dimensiones al diseño de las políticas públicas en términos de complejización de las mismas (Espínola y Silva, 2009; Marcel y Raczynski, 2009; Boisier, 2003). Para fines del artículo, el contenido específico de la expresión complejidad u otras afines remite, por una parte, a la forma que adoptan las consecuencias para el entorno organizacional municipal de los procesos macrosociales de la tecnologización, la profesionalización, la globalización y la creciente presión de los usuarios y de la ciudadanía por obtener mayores servicios. Es decir, al impacto de un conjunto de procesos transversales que presionan los modos tradicionales de funcionamiento del Municipio. La expresión alude, por otro lado, a las nuevas exigencias que moldean las propuestas nacionales de desarrollo en general y en el ámbito de la educación en particular. Es decir, a la búsqueda de soluciones pensadas desde distintas posturas epistemológicas y ámbitos de conocimiento de la realidad social. En efecto, los responsables de diseñar las políticas públicas intentan una síntesis de las dicotomías estructuralistas/ constructivistas, deterministas/relativistas, modernas/post modernas, objetivistas/subjetivistas, entre otras, para abordar de manera más eficiente los problemas de desarrollo (Boisier, 2003).

Las orientaciones multidisciplinarias de las políticas intencionan intervenciones que trascienden los enfoques económicos, asistencialistas o deterministas económicos cuyos gruesos indicadores de éxito cuantificables omiten, usualmente, la multidimensionalidad de la realidad. Asumen la complejidad de los fenómenos sociales que, por décadas, 
separados del desarrollo, han demostrado ser necesarios a la hora de pensar el mejoramiento de la calidad de vida de los sectores postergados. La búsqueda de la integralidad de las actuales propuestas de desarrollo otorgan un rol cada vez más activo a los sujetos en la construcción de su realidad, atendiendo al mismo tiempo a una serie de condiciones como el género, el territorio, la etnicidad, la sustentabilidad y el medio ambiente, entre otras, que antaño simplemente se omitían. De la misma manera, las iniciativas de desarrollo consideran en su diseño estrategias gerenciales emanadas de la economía moderna, a saber: la flexibilidad de las estructuras organizacionales; principios de control de la gestión basados en la transparencia, monitoreo, evaluación y responsabilización de la misma; principios e instrumentos informatizados para las rendiciones cuenta, seguimiento y evaluación de la gestión.

Las nuevas formas de gestión adoptadas por las propuestas de desarrollo demandan cambios substantivos a nivel de las competencias, prácticas y procedimientos y de la cultura organizacional del Municipio, modelando las políticas educacionales. Espínola y Silva (2009), demuestran como la Ley SEP marcó una nueva forma de hacer política educacional al incorporar elementos de control de la gestión, tales como la rendición de cuentas, o la mayor participación de los sostenedores como mediadores de las propuestas en el nivel intermedio. De la misma manera, las Reformas a Ley de educación en el país consideran sistemas de monitoreo y evaluación del desempeño docente complementados con incentivos a aquellos profesionales destacados. Los ejemplos son numerosos pero, más que el detalle, interesa relevar que esta multiplicidad de miradas y de conocimientos disciplinares aplicados al desarrollo dan forma a políticas públicas y programas cada vez más sofisticados, y que demandan a los gobiernos locales competencias cada vez más exigentes paran el desarrollo de la organización municipal.

Se coincide con Zamorano (2006) respecto a que las políticas y programas de mejoramiento educativo dan lugar a un sentimiento de sobre exigencia y a cierta conciencia de algunos funcionarios municipales de que, pese a sus esfuerzos, la situación los supera y no alcanzan el rendimiento óptimo en los Municipios ni escuelas.

Los procesos macrosociales modernizantes señalados -la creciente sofisticación y centralismo de las iniciativas de desarrollo- presionan el entorno municipal que obedece, a la vez, a su propia lógica atravesada por: la rigidez administrativa; el desarrollo incipiente de competencias para asumir la modernización; la orientación hacia el pragmatismo y la contingencia; la tensión entre los intereses políticos y técnicos; la presión constante sobre los escasos recursos municipales y la rotación de las plantas municipales.

Las contraproducentes condiciones estructurales se amplifican en el marco de las crecientes exigencias y cambios que la modernización de las políticas de mejoramiento educativo implica para el Municipio. Consecuentemente, la articulación entre el ritmo de la modernización del Estado -plasmada en el diseño de políticas y programas educacionales- con los límites del sistema organizacional municipal para implementarlas y orientarlas configuran el campo donde operan los actores locales. Las dinámica de las Reformas educacionales se manifiesta en el Municipio por múltiples, sucesivas, diversas, discontinuas y yuxtapuestas propuestas de desarrollo. Éstas presionan e imponen un ritmo acelerado a la organización municipal mediante frecuentes demandas para implementar nuevas políticas, programas, proyectos o acciones educacionales que coexisten, sin que sus objetivos sean siempre complementarios entre sí o correspondientes a las demandas educacionales locales. 
Ahora bien, desde una perspectiva eficientista, acelerar el ritmo de funcionamiento del Municipio e imponer el desarrollo de nuevas y modernas exigencias podría evaluarse positivamente. No obstante, los resultados de la investigación evidencian que esta situación es más bien contraproducente para la calidad de la gestión educacional municipal. Esta lógica de productividad aplicada a lo público estatal, en el contexto de los límites de las capacidades instaladas en los sistemas educacionales públicos, para responder a sus exigencias es definida como "compleja" por lo actores responsables de implementar las políticas y programas educacionales.

En este sentido, la noción de complejidad remite a la vivencia subjetiva de los actores frente a las tendencias de las iniciativas de desarrollo, a su necesidad de que el Municipio les de coherencia y sentido en función de la realidad organizacional. Así queda en evidencia que éste carece de una estrategia consensuada para hacer frente a los cambios de la modernización del Estado, que oriente el conjunto de decisiones que demanda el accionar cotidiano en la implementación de los programas y acciones educacionales. Lo que los funcionarios municipales señalan como: "la falta de una carta de navegación" en la gestión municipal".

La siguiente cita refleja la situación antes señalada:

Nosotros no entendemos pa'dónde va la micro [chilenismo que significa no saber hacia dónde dirigirse], no sabemos realmente, con claridad, ¿Esto es lo que yo quiero para mi gestión? ¿Esto es lo que pretendo hacer? Necesito que vayan todos, se canalice hacia esa dirección, no hay. Cada uno hace su función como les queda más conveniente, de acuerdo a la experiencia que tiene, de acuerdo a los conocimientos que tiene, y así se va sucumbiendo al quehacer diario... ésa es mi percepción, ésa es la realidad" [Participante numero 22, funcionario municipal].

Los funcionarios municipales se refieren a una falta de orientación en la gestión municipal en el quehacer cotidiano sobre el camino trazado por las propuestas, situación favorecida por su falta de participación en el diseño de las mismas. Es más, algunos funcionarios explicitan el vacío de sentido en su quehacer, ocasionado por la lógica mecanicista de aplicación de políticas y programas educacionales. La explicitación de la ambigüedad en la dirección del Municipio y del sistema educativo local, evidencia la falta de referentes normativos, valóricos y técnicos de la labor bien cumplida en función de las necesidades en el contexto socio educacional en el cual trabajan.

Finalmente, se constata que los sistemas educacionales enfrentan un creciente proceso de complejización de su entorno el cual posee dos acepciones: 1) Una refiere a la realidad externa al Municipio. Es decir, a las crecientes y progresivas exigencias en términos de recursos y de competencias de las iniciativas de desarrollo educativo, como también, a la falta de articulación entre las propias iniciativas de desarrollo educativo. 2) Otra, a la realidad interna de la gestión municipal. Esto es, a la falta de articulación entre el ritmo y los requerimientos de las propuestas de mejoramiento educativo y las capacidades del Municipio para implementarlas. Queda igualmente en evidencia la débil orientación en los sistemas de gestión municipal para orientar las prácticas de implementación de las propuestas de desarrollo. El ritmo de exigencias, la falta de articulación entre las propuestas y las capacidades organizacionales y la realidad local se asocian a problemas de coherencia en la gestión y de estabilidad del sistema organizacional. 


\subsection{RESPUESTAS FRENTE A LA COMPLEJIZACIÓN DEL ENTORNO MUNICIPAL}

El conjunto de tensiones que enmarcan la implementación de iniciativas educacionales genera en los funcionarios municipales una lógica operativa conservadora que prioriza el control y la estabilidad de la organización municipal y de los sistemas educacionales locales en desmedro de la calidad técnica de las Reformas.

La lógica pragmática, en el sentido de orientar la acción hacia los productos formales de las políticas, que orienta las prácticas de implementación de las propuestas de mejoramiento educacional constituye una estrategia adaptativa por parte de los funcionarios municipales a las tensiones y dinámica de incidencias con que impactan las Reformas a la organización municipal. Es decir, que es reactiva a la contingencia entendida como condición intrínseca de la centralización. El racional de su conducta se explica para ser más precisos en el privilegio de la estabilidad en el marco de su lectura de la falta de control frente a los procesos de los cuales son parte y frente al escaso manejo que tienen de la incertidumbre.

Los criterios y actitudes con los cuales los funcionarios municipales abordan la implementación de las políticas y programas educacionales responden a la condiciones del contexto organizacional municipal caracterizado por las siguientes tensiones, a saber: 1) Las exigencias y cambios sucesivos de las políticas y programas, 2) La falta de articulación y de continuidad entre las propuestas educacionales que se superponen y yuxtaponen en los sistemas educacionales locales, 3) La dinámica centralizada (verticalista y jerárquica) con la que opera el nivel central para la implementación de las políticas y programas locales 4) La falta de arraigo de las iniciativas de desarrollo educativo con la realidad local 5) La débil orientación de los sistemas de gestión municipal en términos de políticas educacionales locales y a nivel de la organización. Las condiciones anteriormente señaladas llegan en situaciones no poco frecuentes a desbordar las capacidades operativas del Municipio.

De acuerdo a la expresión de un funcionario de DAEM de las comunas en estudio que se presenta, y que refleja la visión del conjunto de actores entrevistados, las Reformas constituyen "un sismo más". Es decir, que son definidas como amenazas externas e incontrolables que pueden producir daño en la organización. ${ }^{10}$

Hoy día estamos con la propuesta del Ministerio de transformar en Corporación de Derecho Público a los Departamentos de Educación, es muy bueno visualizar lo que viene porque nosotros tenemos que ir ajustándonos y preparándonos para ese tema, que puede que pase en cualquier momento, y quisiéramos, que no signifique un sismo más, sino que al contrario, esto pueda seguir en un proceso que no afecte los establecimientos educacionales [Participante numero 19, funcionario municipal].

Se adaptan así a la mecánica sistemática de arribo de las iniciativas valorándolas sólo en la medida que puedan obtener algo "útil”, es decir, o algún beneficio directo objetivable

10 Si bien el análisis de la evidencia empírica demuestra que son sobre todo los funcionarios municipales con más de cinco años de trayectoria quienes priorizan la estabilidad del sistema, no es menos ciertos que otros con menor experiencia comparten la orientación en cuestión. 
de las mismas de acuerdo a su lectura de las necesidades educacionales inmediatas de los sistemas educacionales locales.

Otra situación que ejemplifica el racional de las estrategias pragmáticas de los funcionarios municipales se inscribe la forma en que deciden darle o no prioridad a un programa o proyecto de mejoramiento educativo. Se hace referencia al siguiente escenario: El Ministerio de Educación demanda al Municipio implementar un nuevo programa de mejoramiento educativo. Éste se encuentra aún en la fase piloto de un reciente Programa de Mejoramiento por lo que, en la práctica, la nueva iniciativa coexiste con la anterior, que no es necesariamente complementaria, con la nueva propuesta ni con las necesidades ni capacidades operativas de su sistema educativo local. Los funcionarios municipales se encuentran en la disyuntiva real de tener que decidir: a qué programa darle prioridad, ya que no tienen la capacidad operativa de implementar ambos a cabalidad. Es una disyuntiva de facto, ya que formalmente el Municipio ha de responder a todos los requerimientos del Ministerio de Educación. Las nuevas políticas y programas compiten por los escasos recursos municipales, dónde las dificultades de financiamiento son, aunque con distintos grados urgencia, relevantes para el funcionamiento de la organización (Zamorano 2006). En este contexto, los funcionarios municipales discontinúan el programa en ejecución por falta de presupuesto y de condiciones para operarlos simultáneamente, sobre todo si el financiamiento del gobierno central previsto para la fase piloto del mismo, se encuentra agotado. ${ }^{11}$

De la misma manera, funcionarios municipales afirman que regularmente no hay variaciones substanciales en los Planes de Desarrollo Educativo Anuales Municipales o PADEM, lo que explica porqué los ven como formulismos y requerimientos externos poco útiles para la planificación local ${ }^{12}$, a los cuales responden intentando que no intervengan con su quehacer rutinario. La contradicción aparente es que los PADEM son elaborados por los propios sistemas educacionales locales; aún así no se observa una apropiación de estos instrumentos. De hecho, dentro de las grandes falencias de los instrumentos de planificación es que carecen de suficientes mecanismos de apoyo, seguimiento y control de la gestión planificada.

Los criterios y estrategias pragmáticas con los cuales los funcionarios municipales abordan los requerimientos externos hacen parte de la dinámica operativa intrínseca del Municipio. Siguiendo la misma línea de las ideas, actores testimonian como directores de establecimientos de las comunas en estudio, valoran los programas emblemáticos de mejoramiento de la equidad educacional ${ }^{13}$, por el financiamiento que significan sin referirse explícitamente a su potencial aporte a los procesos de enseñanza aprendizaje. Las políticas y programas adquieren sentido para los actores en la medida que pueden obtener algo útil (concreto o beneficio directo) de acuerdo a su lectura de las necesidades educacionales de los sistemas educacionales locales.

Normalmente estos fondos financian recursos humanos a honorario e insumos.

12 Los instrumentos de planificación son significados por un número importante de funcionarios municipales como dispositivos externos de control disociados, las más de las veces, de la realidad de la organización y de la comuna.

13 Se hace referencia a medidas de mejoramiento educativo orientadas hacia el mejoramiento técnico pedagógico que contemplaban aporte en insumos, equipamiento e infraestructura tales como el P-900, MECE Básica y MECE Media, Red de Informática Educativa, Proyectos de Mejoramiento Educativo (PME), Programa Montegrande y Programa Rural. 
Numerosos ejemplos documentan la lógica práctica con la que los actores locales enfrentan las disyuntivas al momento de llevar a cabo las medidas de desarrollo educativo. Lo importante en este punto de la discusión es dejar en evidencia que se trata de respuestas a la confluencia de tensiones y limitaciones que afectan a los sistemas educacionales locales y centrales y que se acentúa cuanto más foránea sea la política en su desarrollo e implementación. Si bien este ajuste contingente y pragmático de los programas no siempre favorece los objetivos de las propuestas educacionales, significa una forma de adaptación -desde la noción de lógica burocrática- a los efectos contraproducentes de la centralización y a la precariedad de recursos del Sistema Educacional Municipal, que los incita a priorizar el control de la situación (mantención de las rutinas de la vida de la organización) por sobre el aseguramiento de la calidad en la implementación de las iniciativas.

La dinámica anterior tiene como efecto no deseado hacer la organización más impermeable a la realidad social local y a la innovación técnico pedagógico de las Reformas con el objetivo implícito y explicito de disminuir su potencial efecto desestabilizador (distorsión del sistema organizacional) o simplemente por el privilegio que otorga la estabilidad. Privilegio de estabilidad entendido en el sentido weberiano de un racional de construcción de rutinas.

Se sabe que difícilmente se pueden abstraer las conductas de los contextos o campos de acción social (Bourdieu, 1994, 2001), aún si se asume que los actores tienen espacios de indeterminación y que son también responsables de sus acciones (Zemelman, 1992). Se entiende, por la noción de campo, el espacio social dónde se interrelacionan los procesos macrosociales, los determinantes sociales y culturales (capital simbólico y social) que producen y reproducen la acción social. Es allí donde las tensiones del sistema educativo y las lógicas y demandas de las políticas articuladas a la estructura social municipal, condicionan un cierto rango de estrategias, o de prácticas sociales, que van a su vez modelando el escenario y las acciones de los actores. El campo significa límites y posibilidades de acción, es decir, espacios indeterminados y potenciales de construcción de lo posible entendiendo lo posible como acciones que se acercan a las utopías (Zemelman, 1992; Bourdieu, 1999; 2001).

Para los actores Municipales la utopía compartida es el mejoramiento de las condiciones laborales del Municipio a fin de poner a disposición de los jóvenes que habitan su comuna, y particularmente de los más vulnerables, herramientas que les permitan tener una buena educación y, por ende, un futuro mejor. No obstante, el estudio revela que los funcionarios municipales postergan sus ideales en post del la estabilidad de su entorno asociado al cumplimiento funcional de los indicadores cuantitativos y concretos de éxito de las Reformas que les corresponde operar.

El pragmatismo de los líderes intermedios es favorecido por la serie de condiciones transversales o tensiones que complejizan el entorno municipal. Una de éstas es la identataria. Como funcionarios públicos los agentes municipales se adscriben al rol de la cultura organizacional estatal burocrática que supedita las iniciativas de gestión al cumplimiento normativo/operativo. Respecto a la precariedad de la gestión Municipal, ésta se encuentra ampliamente documentada en la literatura. Nos referimos a nudos críticos que refuerzan las estrategias pragmáticas de los líderes intermedios, tales como: la flexibilidad laboral; la rotación de personal; los déficits financieros; la alcaldización de la gestión educativa; y la sobrecarga de trabajo y la politización de la gestión (Espínola 
1997; Lizama y Paredes 2006; Raczynski y Salinas 2006). Se releva, en este contexto, la falta de orientación y de políticas educacionales comunales como uno de los principales factores identificados en el marco del estudio. Otra condición contraproducente con la apropiación de las iniciativas, por parte de los agentes municipales, es la centralización de las iniciativas de desarrollo educativo que refuerza, tal como se había mencionado, el rol de operador de políticas. De la misma manera, su visión crítica respecto a los instrumentos de planificación educativa y comunal los sitúa una vez más como observadores de decisiones externas que llegan al Municipio como requerimientos carentes de sentido orientador de las acciones cotidianas de implementación de las reformas.

Los hallazgos del estudio llevan a concordar con Güell (1998) y Boisier (2003) respecto a que el desarrollo requiere predisposiciones políticas que le den sentido y motiven a quienes las protagonizan. Son justamente las predisposiciones de los funcionarios municipales las que operan en otro sentido, distinto a la búsqueda de la calidad técnica, en la orientación de las prácticas de implementación de políticas y programas educacionales. La acción social estabilizadora o lógica pragmática y utilitaria favorece prescindir de los requerimientos técnico pedagógico de las Reformas a fin de uniformar las prácticas respecto a lo existente, simplificando así su operatoria.

En resumen, se esboza las problemáticas que tensionan la organización municipal y se señala cómo en respuesta a la falta de una clara orientación comunal de las políticas educacionales y de la gestión municipal, así como frente a la precariedad de la misma los actores, despliegan prácticas y se predisponen, conductualmente, hacia la conservación de la estabilidad de los sistemas educacionales locales. Las reacciones conservadoras de los funcionarios locales municipales tienen como efecto perverso no deseado descuidar la calidad técnica de las propuestas de mejoramiento educativo que se implementan en el Municipio ya que se trata de prácticas adaptativas que responden a una racionalidad burocrática operativa.

\section{CONCLUSIONES}

A la luz de los resultados se constata que las dinámicas macrosociales y la estructuración político institucional del sistema educativo, tensionan y desbordan las capacidades de gestión municipal de las comunas semi-urbanas en estudio, condicionando lo que los actores definen como un creciente proceso de complejización del entorno organizacional. En efecto, la articulación entre orientaciones protectoras y neoliberales de las políticas públicas, la precariedad de la descentralización, la disociación entre los componentes del sistema educativo, y la acelerada dinámica y exigencias de la modernización de las reformas, tensionan el contexto organizacional de Municipio. La centralización del país amplifica las distorsiones en la implementación de las políticas y programas educacionales provocados por esta modalidad de funcionamiento, impidiendo el arraigo de las iniciativas de las necesidades, de la realidad y capacidades organizacionales locales.

El Municipio no contaría con un modelo de gestión que le permita compatibilizar los objetivos y requerimientos de las Reformas con los recursos y demandas de los sistemas educacionales locales, particularmente en lo referente a sus orientaciones. Las crecientes exigencias de las políticas y programas educacionales chocan con las débiles directrices locales en el ámbito de la educación y con importantes déficits a nivel de las 
competencias de gestión municipal. La complejización del entorno Municipal se asocia en definitiva a la desestabilización del Sistema Educativo Municipal frente a las dinámicas modernizadoras asociado a la falta de orientación y sinergia entre éste, el nivel educativo central, y las políticas educacionales comunales.

Los actores reaccionan a las controversias del contexto político institucional, las tendencias modernizadoras del sistema educativo y a la precariedad de recursos y del modelo de gestión Municipal, de manera contraria a la cooperación que se espera en el marco de la "buena gobernanza" que orienta las políticas y programas educacionales. Consecuentemente, la gobernanza se objetiviza ${ }^{14}$ en las condiciones que posibilitan su calidad ya que constituye el ámbito amplio en el cual se inscriben las prácticas de implementación de la gestión. En el contexto Municipal, funcionarios definen las políticas y programas de desarrollo como amenazas, desplegando estrategias de implementación cuyo racional es la disminución de la incertidumbre frente al riesgo que representan las Reformas para el equilibrio de la organización. La mantención del equilibrio del sistema Municipal y educacional local en el contexto de las restricciones de recursos, competencias y asimetría entre los ritmos de la organización municipal y de las políticas y programas, favorece la evasión de los aspectos desafiantes e innovadores incorporados en las acciones de mejoramiento. Los funcionarios priorizan y limitan su accionar al cumplimiento de las tareas formales de los políticas y programas que les permiten responder a los gruesos indicadores cuantitativos de éxito requeridos por los sistemas de control de la gestión y de evaluación.

La lógica pragmática que orienta las acciones de desarrollo educativo es avalada por la estructura burocrática del Municipio y la identidad de funcionarios públicos de los actores. Los funcionarios municipales se acomodan así a una situación en la que asumen el menor costo en el contexto de los cambios propuestos por las Reformas. Un efecto contraproducente del pragmatismo es que evidencia la carencia de referentes valóricos normativos y políticos que provean sentido a las prácticas de desarrollo más allá de la lógica funcional exigida externamente por las políticas y programas. La construcción de sentido de las prácticas aporta eficiencia a la tarea ya que moviliza recursos motivacionales subjetivos, genera sinergia en la interacción social y permite la apropiación e identificación con las políticas y programas de desarrollo educativo.

El éxito de las Reformas Educacionales dirigidas a los niveles locales no sólo debe flexibilizar sus objetivos y metas en función de los diagnósticos educacionales comunales, sino que también cuidar su estabilidad organizacional. El resguardo de los equilibrios organizacionales implica: 1) Adecuar el ritmo de implementación de las propuestas de desarrollo en función de las capacidades y potencialidades de gestión municipal. 2) La construcción significativa a partir de la visión de los funcionarios Municipales del sentido y de la proyección en el territorio de las propuestas de mejoramiento educativo. 3) Desarrollar los procesos para que los Funcionarios Municipales adquieran las capacidades que no disponen y que les permitan ser protagonistas de los objetivos de mejoramiento de las políticas y programas educativos comunales.

14 Entendemos la objetivización como las manifestaciones externas (concretas o "reales") de los fenómenos y/o de sus dimensiones abstractas. 


\section{REFERENCIAS BIBLIOGRÁFICAS}

Auguste, S. y Valenzuela, J.P. (2004). Do Students Benefit from School Competition? The Chilean Experience. Tesis de Grado University of Michigan. Buenos Aires: Centro de Estudios de las Políticas Públicas (CEPP) /Ministerio de Educación, Cultura y Tecnología de la República Argentina.

Atria, F. (2010). ¿Qué es la educación "pública"? En C. Bellei, J. P. Valenzuela (eds.), Ecos de la revolución pingüina: avances, debates y silencios en la Reforma educacional (pp. 153-181). Santiago, Universidad de Chile Unicef.

Avellaneda, C. N. (2009). Municipal Performance: Does Mayoral Quality Matter? Journal of Public Administration Research and Theory, vol. 19, n. 2, 285-312.

Bellei, C. y González, P. (2003). Educación y Competitividad en Chile. Documento de Trabajo, Santiago de Chile: Facultad Latinoamericana de Ciencias Sociales.

Boisier, S. (2005). Crónica de una muerte frustrada: el territorio en la globalización. La recuperación de las políticas territoriales. Politika. Revista de Ciencias Sociales, vol. 1, 11-25. (2003). ¿Y si el desarrollo fuese una emergencia sistémica? Ciudad y territorio: Estudios territoriales, vol. 138, 565-588.

Bourdieu, P. (1994). Raisons pratiques. Sur la théorie de l'action. Paris: Raisons d'agir. (2001). Science de la science et réflexivité. Paris: Raisons d'agir.

GOBIERNO DE CHILE (2009). Texto actualizado / Diciembre 2009. Ley N $N^{o} 19.175$, Orgánica Constitucional Sobre Gobierno y Administración Regional y Jurisprudencia Administrativa, Ministerio del Interior. Subsecretaría de Desarrollo Regional y Administrativo Santiago: Gobierno de Chile.

Castillo, P. (2006). El desarrollo local en la gestión municipal. Ciencias Sociales, vol. III, n. 1, 103-114. Disponible en: http://www.uvm.cl/csonline/2006_1/pdf/desarrollolocal.pdf

Caro, C. (2005). Resultados SIMCE 2002. Derribando Mitos. Santiago: Departamento de Estudios Municipales de la División de Políticas y Estudios. Subsecretaría de Desarrollo Regional y Administrativo.

Castro, M. (2010). Códigos para el Análisis de Política Educativa Local en Chile: Temas Pendientes. Revista Ensaio Pesquisia para la eduçao em ciencias, vol 18, n. 67, 189-213. Consultado15-09-2010 En Scielo Revista Ensaio: http://www.portal.fae.ufmg.br/seer/index. php/ensaio.

Cox, C. y Lemaitre, M.J. (1999). Market y State Principles of Reform in Chile Education: Policies y Results. En Chile: Recent Policy Lessons y Emerging Challenges. (ed.). Washington D.C.: WDI Development Studies Series.

Di Gropello, E. (1999). Los modelos de descentralización educativa en América Latina. Revista de la Cepal 68, 154-170.

Donoso S., Hawes, G. (2002). Eficiencia escolar y diferencias socioeconómicas: a propósito de los resultados de las pruebas de medición de la Calidad de la Educación en Chile. Educação e Pesquisa, vol. 28, n. 2: 25-40.

Donoso, S. (2005). Reforma y política educacional en Chile 1990-2004: El neoliberalismo en crisis. Revista Estudios Pedagógicos, vol. 31, (1). Consultado el 04 de Octubre de 2006 en World Wide En Estudios Pedagógicos: http://mingaonline.uach.cl/scielo.php?script=sci_arttext\&pid= S071807052005000100007\&lng=es \&nrm=iso>. ISSN 0718-0705.

Donoso, S. (2008) La política de financiamiento de la Educación básica en Chile 1990-2008: consecuencias y disyuntivas. Jornal de Políticas Educacionais vol. 3, n. 17-29.

Donoso, S. y Donoso. G. (2009) Políticas de Gestión de la Educación Pública Escolar en Chile (1990-2010): Una evaluación inicial. Ensaio 64, vol. 17, 412 -448. (2008). Consecuencias y disyuntivas. Jornal de Políticas Educacionais 3, 17-29. 
Donoso, S., Arias O., Cancino V., Oyarzun C. (2010). Los Fundamentos de la Intervención. Desarrollo educativo al servicio del desarrollo social. En J. G. y. D. Castro, Proyecto de apoyo y fortalecimiento educacional en gestión directiva y competencias profesionales docentes (pp. 69-90). FIDECAP.

Eyzaguirre, N., Marcel, M. Rodríguez, J. y Tokman, M. (2005). Hacia la economía del conocimiento: El camino para crecer con equidad en el largo plazo. Estudios Públicos, n. 97, 5-57.

Elacqua, G. y Fabrega, R. (2007). El consumidor de la educación: El actor olvidado elección de escuelas en Chile. En S. Cuesto (ed.), Uso e impacto de la información educativa en América Latina. Santiago, Chile: PREAL.

Espínola, V. (1997). Descentralización del sistema educativo en Chile: impacto en la gestión de las escuelas. Grupos de Desarrollo Humano. Región de América Latina y el Caribe, n. 10.

Espínola, V. y Silva, M.E. (2009). Competencias del sostenedor para una efectiva gestión del mejoramiento educativo en el nivel local: Una propuesta Expansiva. Santiago: UDP, CIEPLAN 34.

Espínola, V., Chaparro, M., Guzmán, A., Gálvez, R. y Lazcano, L. (1996). Transformaciones en los procesos de descentralización: El caso de Chile. Santiago: CIDE.

FLACSO-CHILE (2008). Modernizar el Estado: hacia un Chile Desarrollado y Competitivo. Disponible en http://www.modernizacion.cl/filesapp/flasco_modernizacion. Visitado el 20-10-2010.

Giddens, A. y Hutton, W. (2000). On The Edge. Living with Global Capitalism. London: Vintage. Guell, P. (1999). Subjetividad social y Desarrollo Humano. Mimeo.

Fuentes, L., Allard, P. y OrellanA, A. (2007). El Municipio y la gobernabilidad del territorio comunal. En La Reforma Municipal en la Mira. Identificando los Municipios prioritarios en la Región Metropolitana: Complejidad comunal versus condiciones para la calidad de la gestión municipal. Santiago de Chile: Expansiva.

Gallego, F. y Seebach, C. (2007). Indicadores de complejidad y resultado en el sector de educación municipal. La Reforma Municipal en la Mira Identificando los Municipios prioritarios en la Región Metropolitana: Complejidad comunal versus condiciones para la calidad de la gestión municipal. Santiago de Chile: Expansiva.

Garcia-Huidobro, J.E. y COX, C. (1999). La Reforma Educacional Chilena 1990 -1998. Visión de Conjunto. En J. E. García-Huidobro (ed.), La Reforma Educacional Chilena (pp. 7-46). Madrid: Popular.

González, P., Mizala, A. y Romaguera, P. (2002). Recursos diferenciados a la educación subvencionada en Chile. Serie Economía, vol. 150, 1-42.

Firinguetti, Luis and Pavez, Ricardo (2008) Inequidades en la Enseñanza Media Municipalizada: El caso de la Provincia de Concepción. Última década, vol.16, n.29, 49-70.

CHILE, M. D. E. G. D. (2009). SIMCE M. D. EDUCACION. Santiago de Chile, Gobierno de Chile. Consultado en Gobierno de Chile, Ministerio de Educación.

Hoxby, C. (2002). Would School Choice Change the Teaching Profession? The Journal of Human Resources, n. 37, 846-891.

Kooiman, J., ed. (1993). Modern Governance: New Government-Society Relations. Londres: Sage. Lizama, O. y Paredes, R. (2006). Restricciones, Gestión y Brecha Educativa en Escuelas Municipales. Santiago: Politeia Universidad Católica de Chile.

(2008). Estudio Mejoramiento de la Gestión y la Calidad de la Educación Municipal. Informe Ejecutivo.

Raczynski, D. y Salinas, D. (2006). La Educación en el Nivel Local: ¿Cómo Manejar la Tensión Entre lo Técnico y lo Político? Consultado 22-06-2010 En: http://educaciondec.cl/gestion/wpcontent/uploads/2009/09/La-educación-en-el-nivel-local.pdf

Larrañaga, O. (1995). "Descentralización de la Educación en Chile: Una Evaluación Económica*." Estudios Públicos, n. 60: 2-43. 
Román, M., Y Carrasco, A. (2007). Los niveles intermedios del sistema escolar chileno: posibilidades y limitaciones para la equidad. REICE, vol. 5, n. 1, 1-21.

Marcel, M. y Raczynski, D. (Ed.) (2009). La Asignatura Pendiente. Claves para la revalidación de la Educación Pública de Gestión Local en Chile. Santiago de Chile: Uqbar.

Moe, T. (2001). Schools, Vouchers, and the American Public. Washington D.C.: The Brookings Institution Press.

Matus, T., Ibarra, R., Fuentes, L. y Pérez, U. (2007). La Reforma Municipal en la Mira Identificando los Municipios prioritarios en la Región Metropolitana: Complejidad comunal versus condiciones para la calidad de la gestión municipal. Expansiva. Observatorio de Ciudades y Escuela de Trabajo Social de la Pontificia Universidad Católica de Chile las políticas públicas. n. 26. Consultado 30-01-2010. Disponible http://www.expansiva.org/media/archivos/20071109111558. pdf

Monje-Reyes, P. (2007). El Municipio y su aporte a la implementación y desarrollo de políticas sociales intersectoriales: una visión crítica de la experiencia chilena, Reforma del Estado y de la Administración Pública. XII Congreso Internacional. Stgo. Domingo, Rep. Dominicana: CLAD.

Mons, N. (2007). Les Nouvelles Politiques éducatives.Paris: PUF.

Ocde (2003). Informe de antecedentes del país para OCDE: evaluación de las políticas educacionales de Chile. Paris: OCDE.

-(2004). Chile. Revisión de las políticas Nacionales de Educación. París: Organización para la Cooperación y el Desarrollo.

-(2009). Chile. Revisión de las políticas Nacionales de Educación. París: Organización para la Cooperación y el Desarrollo..

-(2010). Revisión Económica Chile 2010. París: Organización para la Cooperación y el Desarrollo.

Oliva, M.A. (2010). La política de la educación chilena 1965-2009. ¿Qué oculta esa trama? Revista Brasileña de Educación, vol. 15, n. 44, 311-328.

Osborne, D. y Gaebler, T. (1994). La reinvención del gobierno. Barcelona: Paidós.

Porter, M. (1998). On Competition. Boston: Harvard Business School.

Paredes, R. y Lizama, O. (2006). "Restricciones, Gestión y Brecha Educativa en Escuelas Municipales". Documento Centro de Políticas.

Pavez, M.A. (2004). Municipios Efectivos en Educación. Santiago: Universidad de Chile.

Plumptre, J. y Graham J. (1999). Governance and Good Governance: International and Aboriginal Perspectives. Institute of Governance.

Quezada-Hofflinger, A. (2009). Juntos Pero no Revueltos: Desigualdad Económica y Educacional en Chile. Texas: The University of Texas Inequality Project.

Raczynski, D. y Salinas, D. (2007). La Educación en el Nivel Local: ¿Cómo manejar la Tensión entre lo Técnico y lo Político?, Santiago de Chile: CIEPLAN/Asesorías para el desarrollo. n. 3.

Serrano, C. (2001). Inversión pública y gestión regional: Nudos críticos. En Racsynki, D., Serrano, C. (eds.), Descentralización: Nudos críticos. Santiago de Chile: CIEPLAN/Asesorías para el desarrollo.

Sapelli, C. (2003). Introducción: La Economía de la Educación y el Sistema Educativo Chileno. Cuadernos de Economía, n. 118, 281-296.

Sapelli, C. y B. Vial (2002). The performance of private and public school in the Chilean voucher system. Cuadernos de Economía, n. 118, 423-454.

Whittingham, M. V. (2002). "Aportes de la teoría y la praxis para la nueva gobernanza." VII Congreso Internacional del CLAD sobre la Reforma del Estado y de la Administración Pública, Lisboa, 8-11 oct.

Wong, K. (1992). Choice in Public Schools: Their Institucional Functions and Distributive Consequences. Research in Urban Policy, vol. 4, 175-98. 
Zamorano, L. (2006). Descentralización De La Educación y El Desempeño de Los Municipios. Santiago: Instituto Chileno de Estudios Municipales/Universidad Autónoma de Chile.

Zemelman, H. (1992). Horizontes de la Razón I y II. Barcelona: Anthropos. 
